\title{
NORMAL SEQUENCES WITH GIVEN LIMITS OF MULTIPLE ERGODIC AVERAGES
}

\author{
Lingmin LiaO AND Michą RAmS
}

\begin{abstract}
We are interested in the set of normal sequences in the space $\{0,1\}^{\mathbb{N}}$ with a given frequency of the pattern 11 in the positions $k, 2 k$. The topological entropy of such sets is determined.
\end{abstract}

2010 Mathematics Subject Classification: Primary: 28A80; Secondary: 11K16, 37B40.

Key words: normal sequences, topological entropy, multiple ergodic averages, Hausdorff dimension.

\section{Introduction and statement of results}

Recently, Fan, Liao, and Ma ([5]), and Kifer ([10]) proposed to calculate the topological entropy spectrum of level sets of multiple ergodic averages. Here, the topological entropy means Bowen's topological entropy (in the sense of [3]; see the definition in Section 2) which can be defined for any subset, not necessarily invariant or closed.

Let $\Sigma=\{0,1\}^{\mathbb{N}}$. Among other questions, Fan, Liao, and Ma ([5] $)$ asked what is the topological entropy of

$$
A_{\alpha}:=\left\{\left(\omega_{k}\right)_{1}^{\infty} \in \Sigma: \lim _{n \rightarrow \infty} \frac{1}{n} \sum_{k=1}^{n} \omega_{k} \omega_{2 k}=\alpha\right\} \quad(\alpha \in[0,1]) .
$$

As a first step to solve the problem, they also suggested to study a subset of $A_{0}$ :

$$
A:=\left\{\left(\omega_{k}\right)_{1}^{\infty} \in \Sigma: \omega_{k} \omega_{2 k}=0 \text { for all } k \geq 1\right\} .
$$

The topological entropy of $A$, denoted by $h_{\text {top }}(A)$, was later given by Kenyon, Peres, and Solomyak [9].

Theorem 1.1 (Kenyon-Peres-Solomyak). We have

$$
h_{\text {top }}(A)=-\log (1-p)=0.562399 \ldots,
$$

where $p$ is the unique real solution in $[0,1]$ of

$$
p^{2}=(1-p)^{3} .
$$


Building on the idea of [9], the question about the topological entropy of $A_{\alpha}$ was finally answered by Peres and Solomyak [12], and then in higher generality by Fan, Schmeling, and $\mathrm{Wu}[\mathbf{6}]$.

Theorem 1.2 (Peres-Solomyak, Fan-Schmeling-Wu). For any $\alpha \in[0,1]$, we have

$$
h_{\mathrm{top}}\left(A_{\alpha}\right)=-\log (1-p)-\frac{\alpha}{2} \log \frac{q(1-p)}{p(1-q)},
$$

where $(p, q)$ is the unique solution in $[0,1]^{2}$ of the system

$$
\left\{\begin{array}{l}
p^{2}(1-q)=(1-p)^{3}, \\
2 p q=\alpha(2+p-q) .
\end{array}\right.
$$

In particular, $h_{\mathrm{top}}\left(A_{0}\right)=h_{\mathrm{top}}(A)$.

Another interesting related set is

$$
B:=\left\{\left(\omega_{k}\right)_{1}^{\infty} \in \Sigma: \omega_{k}=\omega_{2 k} \text { for all } k \geq 1\right\} .
$$

A sequence $\omega \in\{0,1\}^{\mathbb{N}}$ is said to be simple normal if the frequency of the digit 0 in the sequence is $1 / 2$. It is said to be normal if, for all $n \in \mathbb{N}$, each word in $\{0,1\}^{n}$ has frequency $1 / 2^{n}$. We denote the set of normal sequences by $\mathcal{N}$.

We are interested in the intersection of $\mathcal{N}$ with the set $A_{\alpha}$ of given frequency of the pattern 11 in $\omega_{k} \omega_{2 k}$. For the usual ergodic (Birkhoff) averages the normal sequences all belong to one set in the multifractal decomposition - the situation for multiple ergodic averages turns out to be very different.

Our results are as follows:

Theorem 1.3. For $\alpha \leq 1 / 2$ we have

$$
h_{\text {top }}\left(\mathcal{N} \cap A_{\alpha}\right)=\frac{1}{2} \log 2+\frac{1}{2} H(2 \alpha),
$$

where $H(t)=-t \log t-(1-t) \log (1-t)$. For $\alpha>1 / 2$, the set $\mathcal{N} \cap A_{\alpha}$ is empty.

Further,

$$
h_{\text {top }}(\mathcal{N} \cap A)=h_{\text {top }}\left(\mathcal{N} \cap A_{0}\right)=\frac{1}{2} \log 2 .
$$

Moreover, $\mathcal{N} \cap B \subset A_{1 / 2}$ and

$$
h_{\text {top }}(\mathcal{N} \cap B)=h_{\text {top }}\left(\mathcal{N} \cap A_{1 / 2}\right)=h_{\text {top }}(B)=\frac{1}{2} \log 2 .
$$


The last statement of Theorem 1.3 was recently proved, in higher generality, by Aistleitner, Becher, and Carton [1] .

Let us now define the set of sequences with prescribed frequency of 0 's and 1's:

$$
E_{\theta}:=\left\{\omega \in \Sigma: \lim _{n \rightarrow \infty} \frac{\omega_{1}(\omega)+\cdots+\omega_{n}(\omega)}{n}=\theta\right\} .
$$

In particular, $E_{1 / 2}$ is the set of simple normal sequences.

Theorem 1.4. We have

$$
h_{\mathrm{top}}\left(E_{\theta} \cap A_{\alpha}\right)=\left(1-\frac{\theta}{2}\right) H\left(\frac{2 \theta-\alpha}{2-\theta}\right)+\frac{\theta}{2} H\left(\frac{\theta-\alpha}{\theta}\right)
$$

for $\alpha \leq \theta \leq(2+\alpha) / 3$, otherwise $E_{\theta} \cap A_{\alpha}=\emptyset$. Further,

$$
h_{\text {top }}\left(E_{\theta} \cap A\right)=h_{\text {top }}\left(E_{\theta} \cap A_{0}\right)=\frac{2-\theta}{2} H\left(\frac{2 \theta}{2-\theta}\right) .
$$

Note that

$$
h_{\text {top }}\left(E_{1 / 2} \cap A\right)=\frac{3}{4} H\left(\frac{2}{3}\right)>h_{\text {top }}(\mathcal{N} \cap A) .
$$

Applying the results of $[\mathbf{1 2}]$, we have the following corollary.

Corollary 1.5. The equality

$$
h_{\text {top }}\left(E_{\theta} \cap A_{\alpha}\right)=h_{\text {top }}\left(A_{\alpha}\right)
$$

holds if and only if $\alpha$ and $\theta$ satisfy the relation

$$
(2 \theta-\alpha)^{2}(\theta-\alpha)(2-\theta)=\theta(2-3 \theta+\alpha)^{3} .
$$

In particular, when

$\theta=\frac{2}{3}\left(1+\left(\frac{2}{23}\right)^{2 / 3} \sqrt[3]{3 \sqrt{69}-23}-\left(\frac{2}{23}\right)^{2 / 3} \sqrt[3]{3 \sqrt{69}+23}\right)=0.354 \ldots$,

i.e., the unique real solution of the equation $4 \theta^{2}(2-\theta)=(2-3 \theta)^{3}$, we have

$$
\operatorname{dim}_{H} E_{\theta} \cap A=\operatorname{dim}_{H} A .
$$

We organize our paper as follows. In Section 2 we give some preliminaries. Section 3 is devoted to the proof of Theorem 1.3. The proofs of Theorem 1.4 and Corollary 1.5 are given in Section 4. 


\section{Preliminaries}

2.1. Bowen's topological entropy. In 1973, Bowen ([3]) introduced a definition of topological entropy for any subset which is not necessarily invariant or closed. Though the original definition of Bowen's topological entropy is for any topological dynamical systems we recall, for simplicity, the definition of Bowen's entropy for a topological dynamical system $(X, T)$ equipped with a metric $d$. For $x \in X, n \in \mathbb{N}, n \geq 1$, denote by $B_{n}(x, \varepsilon)$ the Bowen ball defined by

$$
B_{n}(x, \varepsilon):=\left\{y \in X: d\left(T^{k} x, T^{k} y\right)<\varepsilon, \forall k=0, \ldots, n-1\right\} .
$$

For $E \subset X, s \geq 0, N \geq 1$, and $\varepsilon>0$, set

$$
\mathcal{H}_{N}^{s}(E, \varepsilon)=\inf \sum_{i} \exp \left(-s n_{i}\right),
$$

where the infimum is taken over all finite or countable families $\left\{B_{n_{i}}\left(x_{i}, \varepsilon\right)\right\}$ such that $x_{i} \in X, n_{i} \geq N$, and $E \subset \bigcup_{i} B_{n_{i}}\left(x_{i}, \varepsilon\right)$. The quantity $\mathcal{H}_{N}^{s}(E, \varepsilon)$ is non-decreasing as $N$ increases, so the following limit exists

$$
\mathcal{H}^{s}(E, \varepsilon)=\lim _{N \rightarrow \infty} \mathcal{H}_{N}^{s}(E, \varepsilon) .
$$

For the quantity $\mathcal{H}^{s}(E, \varepsilon)$ considered as a function of $s$, there exists a critical value, which we denote by $h_{\mathrm{top}}(E, \varepsilon)$, such that

$$
\mathcal{H}^{s}(E, \varepsilon)= \begin{cases}+\infty, & s<h_{\text {top }}(E, \varepsilon), \\ 0, & s>h_{\text {top }}(E, \varepsilon) .\end{cases}
$$

One can prove that the following limit exists

$$
h_{\mathrm{top}}(E)=\lim _{\varepsilon \rightarrow 0} h_{\mathrm{top}}(E, \varepsilon) .
$$

The quantity $h_{\text {top }}(E)$ is called the topological entropy of $E$.

We remark that for the symbolic dynamical system $(\Sigma, \sigma)$, where the space $\Sigma=\{0,1\}^{\mathbb{N}}$ is equipped with the usual metric defined by

$$
\forall \omega, \tau \in \Sigma, \quad d(\omega, \tau):=2^{-\min \left\{n \geq 0: \omega_{n+1} \neq \tau_{n+1}\right\}},
$$

and $\sigma$ is the left shift defined by

$$
\sigma: \omega_{1} \omega_{2} \ldots \mapsto \omega_{2} \omega_{3} \ldots,
$$

the Bowen ball $B_{n}(\omega, \varepsilon)(\varepsilon<1)$ is nothing but the cylinder $C_{n}\left(\omega_{1}, \ldots, \omega_{n}\right)$ of order $n$ defined by

$$
C_{n}\left(\omega_{1}, \ldots, \omega_{n}\right):=\left\{\tau \in \Sigma: \tau_{1}=\omega_{1}, \ldots, \tau_{n}=\omega_{n}\right\},
$$


and Bowen's topological entropy $h_{\text {top }}(E)$ and Hausdorff dimension of a subset $E \subset X, \operatorname{dim}_{H}(E)$, differ only by a constant:

$$
h_{\text {top }}(E)=(\log 2) \cdot \operatorname{dim}_{H}(E) .
$$

We refer to Falconer's book [4] for the details on Hausdorff dimension.

2.2. Billingsley's lemma for Bowen's entropy. The Mass Distribution Principle ([4, Principle 4.2]) or, more generally, Billingsley's lemma ([2]) for the Hausdorff dimension has the following topological entropy version $([\mathbf{1 1}])$.

Let $\mu$ be a Borel probability measure on $X$. The local entropy $h_{\mu}(x)$ at a point $x \in X$ is defined as

$$
h_{\mu}(x)=\lim _{\varepsilon \rightarrow 0} \liminf _{n \rightarrow \infty} \frac{-\log \mu\left(B_{n}(x, \varepsilon)\right)}{n} .
$$

Theorem 2.1 (Ma-Wen, 2008). Let $\mu$ be a Borel probability measure on $X, E \subset X$ be a Borel subset, and $0<h<\infty$. Then

(i) if $h_{\mu}(x) \leq h$ for all $x \in E$, then $h_{\text {top }}(E) \leq h$,

(ii) if $h_{\mu}(x) \geq h$ for all $x \in E$ and $\mu(E)>0$, then $h_{\text {top }}(E) \geq h$.

We remark that in the symbolic dynamical system $(\Sigma, \sigma)$, the local entropy $h_{\mu}(\omega)$ at a point $\omega \in \Sigma$ is

$$
h_{\mu}(\omega)=\liminf _{n \rightarrow \infty} \frac{-\log \mu\left(C_{n}(\omega)\right)}{n},
$$

where $C_{n}(\omega)$ is the cylinder of order $n$ containing the point $\omega$.

2.3. A lemma of elementary analysis. The following lemma of Peres and Solomyak ([12, Lemma 5]) will be applied several times.

Lemma 2.2 (Peres-Solomyak). Suppose that $\left\{z_{n}\right\}$ is a bounded real sequence and there exists $c>0$ such that $\left|z_{n}-z_{n+m}\right| \leq c \frac{m}{n}$ for all $m, n \in$ $\mathbb{N}$. If $z_{2^{k} n} \rightarrow \gamma$ as $k \rightarrow \infty$ for all $n \in \mathbb{N}$, then $z_{n} \rightarrow \gamma$.

2.4. A family of measures on $\{\mathbf{0 , 1}\}^{\mathbb{N}}$. For the lower bound estimations of the topological entropy, the following family of measures on $\{0,1\}^{\mathbb{N}}$ will be used. Let $\left(p_{0}, p_{1}\right)$ be a probability vector, i.e., $p_{0}, p_{1} \geq$ 0 and $p_{0}+p_{1}=1$. Let

$$
\left(\begin{array}{ll}
p_{00} & p_{01} \\
p_{10} & p_{11}
\end{array}\right)
$$

be a transition matrix with all coefficients $p_{i j} \geq 0$ and $p_{i 0}+p_{i 1}=1$ for $i=0,1$. We also assume the following condition which will guarantee our measures to be non-trivial:

$$
\left|p_{01}-p_{11}\right| \neq 1 \text {. }
$$


With the data $p_{i}, p_{i j}(i, j \in\{0,1\})$, we define a Borel measure $\mu$ on the space $\Sigma=\{0,1\}^{\mathbb{N}}$ as follows

- if $k$ is odd, then $\omega_{k}=1$ with probability $p_{1}$,

- if $k$ is even and $\omega_{k / 2}=1$, then $\omega_{k}=1$ with probability $p_{11}$,

- if $k$ is even and $\omega_{k / 2}=0$, then $\omega_{k}=1$ with probability $p_{01}$,

with the events $\left\{\omega_{k}=1\right\}$ and $\left\{\omega_{\ell}=1\right\}$ independent except when $k / \ell$ is a power of 2 . More precisely, we define the measure $\mu$ on any cylin$\operatorname{der} C_{n}\left(\omega_{1} \cdots \omega_{n}\right)$ of order $n \in \mathbb{N}$ by

$$
\mu\left(C_{n}\left(\omega_{1} \cdots \omega_{n}\right)\right)=\prod_{k=1}^{\lceil n / 2\rceil} p_{\omega_{2 k-1}} \cdot \prod_{k=1}^{\lfloor n / 2\rfloor} p_{\omega_{k} \omega_{2 k}}
$$

where $\lceil\cdot\rceil,\lfloor\cdot\rfloor$ denote the ceiling function and the integer part function respectively. Then by Kolmogorov consistence theorem, $\mu$ is well defined on $\Sigma$. We remark that the measure $\mu$ depends on the given data $p_{i}, p_{i j}$ $(i, j \in\{0,1\})$. We will see later that by suitably choosing these data, we can find suitable measures supported on the sets. Such measures are essential for calculating the topological entropy of the sets.

For $\omega \in \Sigma$ and $n \in \mathbb{N}$, set

$$
x_{n}(\omega)=\frac{2}{n} \sum_{k=n / 2+1}^{n} \omega_{k} .
$$

The following Lemmas 2.3, 2.6, and 2.9 will be useful.

Lemma 2.3. For $\mu$-almost all $\omega$, as $n \rightarrow \infty$,

$$
x_{2 n}(\omega)=\frac{1}{2} p_{1}+\frac{x_{n}(\omega)}{2} p_{11}+\frac{1-x_{n}(\omega)}{2} p_{01}+o(1) .
$$

Proof: Recall that, by the definition of the measure $\mu$, the random variables $\omega_{k}$ and $\omega_{\ell}$ are independent except when $k / \ell$ is a power of 2 . Note that $x_{n}(\omega)$ is the average of $\omega_{k}$ 's where no two different $k$ 's have a quotient that is a power of 2 . Thus for those $n / 2+1 \leq k \leq n, \omega_{k}$ are independent. Thus by Hoeffding's inequality ([7, Theorem 1]), we have

$$
\mu\left(\left|x_{2 n}-\mathbb{E}_{\mu}\left(x_{2 n} \mid x_{n}\right)\right|>n^{-1 / 4}\right) \leq e^{-2 \sqrt{n}} .
$$

To calculate $\mathbb{E}_{\mu}\left(x_{2 n} \mid x_{n}\right)$. Let us consider the expected numbers of $\{k \in$ $\left.(n / 2, n]: \omega_{2 k-1}=1\right\}$, of $\left\{k \in(n / 2, n]: \omega_{k}=1 \wedge \omega_{2 k}=1\right\}$, and of $\left\{k \in(n / 2, n]: \omega_{k}=0 \wedge \omega_{2 k}=1\right\}$ under the condition of $x_{n}$. The first one is not really conditional: it is equal to $p_{1} \cdot n / 2$. The second equals $p_{11} \cdot \sharp\left\{k \in(n / 2, n]: \omega_{k}=1\right\}=p_{11} \cdot x_{n} \cdot n / 2$. The third equals $p_{01}$. $\sharp\left\{k \in(n / 2, n]: \omega_{k}=0\right\}=p_{01} \cdot\left(1-x_{n}\right) \cdot n / 2$. By noting that under the 
condition of $x_{n}$, the value of $x_{2 n}$ is the sum of the above three numbers divided by $n$, we have

$$
\mathbb{E}_{\mu}\left(x_{2 n} \mid x_{n}\right)=\frac{1}{2} p_{1}+\frac{x_{n}}{2} p_{11}+\frac{1-x_{n}}{2} p_{01} .
$$

Hence, by (2.2) and (2.3), we have

$$
\mu\left(\left|x_{2 n}(\omega)-\left(\frac{1}{2} p_{1}+\frac{x_{n}(\omega)}{2} p_{11}+\frac{1-x_{n}(\omega)}{2} p_{01}\right)\right|>n^{-1 / 4}\right) \leq e^{-2 \sqrt{n}} .
$$

Using the summability of the series $e^{-2 \sqrt{n}}$ and applying the BorelCantelli Lemma, we complete the proof.

Applying Lemma 2.2, we can determine the $\mu$-almost sure limit of $x_{n}(\omega)$.

Corollary 2.4. For $\mu$-almost all $\omega$,

$$
\lim _{n \rightarrow \infty} x_{n}(\omega)=\xi:=\frac{p_{1}+p_{01}}{2-p_{11}+p_{01}} .
$$

Proof: Note that by condition (2.1),

$$
\left|\frac{p_{01}-p_{11}}{2}\right|<1
$$

Thus by Lemma 2.3, $\mu$-almost surely, as $k \rightarrow \infty$,

$$
x_{2^{k} n}(\omega) \rightarrow \frac{p_{1}+p_{01}}{2-p_{11}+p_{01}} .
$$

By Lemma 2.2, this implies that $\mu$-almost surely

$$
\lim _{n \rightarrow \infty} x_{n}(\omega)=\frac{p_{1}+p_{01}}{2-p_{11}+p_{01}} .
$$

Proposition 2.5. For $\mu$-almost all $\omega$,

$$
\lim _{n \rightarrow \infty} \frac{1}{n} \sum_{j=1}^{n} \omega_{j}=\xi=\frac{p_{1}+p_{01}}{2-p_{11}+p_{01}}
$$

Proof: By Corollary 2.4, for $\mu$-almost all $\omega$, for all $n$,

$$
\lim _{k \rightarrow \infty} \frac{1}{2^{k} n} \sum_{j=1}^{2^{k} n} \omega_{j}=\lim _{k \rightarrow \infty} \frac{1}{2^{k} n}\left(\sum_{i=1}^{k} 2^{i-1} n \cdot x_{2^{i} n}(\omega)+\sum_{j=1}^{n} \omega_{j}\right)=\xi .
$$

Applying Lemma 2.2, we have

$$
\lim _{n \rightarrow \infty} \frac{1}{n} \sum_{j=1}^{n} \omega_{j}=\xi, \quad \mu \text {-a.e. }
$$


Lemma 2.6. We have, for $(i, j) \in\{0,1\}^{2}$, for $\mu$-almost all $\omega$, as $n \rightarrow \infty$,

$$
\frac{2}{n} \sum_{k=n / 2+1}^{n} \mathbf{1}_{\left\{\omega_{k} \omega_{2 k}=i j\right\}}(\omega)=\left(\frac{2}{n} \sum_{k=n / 2+1}^{n} \mathbf{1}_{\left\{\omega_{k}=i\right\}}(\omega)\right) \cdot p_{i j}+o(1),
$$

and in particular,

$$
\frac{2}{n} \sum_{k=n / 2+1}^{n} \omega_{k} \omega_{2 k}=x_{n}(\omega) \cdot p_{11}+o(1) \quad(n \rightarrow \infty) .
$$

Proof: Note that for $n / 2+1 \leq k \leq n$, the variables $\omega_{k} \omega_{2 k}$ are independent. Then the same argument as in the proof of Lemma 2.3 gives us the assertion.

By Lemma 2.6 and Corollary 2.4, we immediately obtain the following corollary.

Corollary 2.7. We have, for $j \in\{0,1\}$, for $\mu$-almost all $\omega$,

$$
\lim _{n \rightarrow \infty} \frac{2}{n} \sum_{k=n / 2+1}^{n} \mathbf{1}_{\left\{\omega_{k} \omega_{2 k}=0 j\right\}}(\omega)=(1-\xi) \cdot p_{0 j}
$$

and

$$
\lim _{n \rightarrow \infty} \frac{2}{n} \sum_{k=n / 2+1}^{n} \mathbf{1}_{\left\{\omega_{k} \omega_{2 k}=1 j\right\}}(\omega)=\xi \cdot p_{1 j},
$$

and in particular,

$$
\lim _{n \rightarrow \infty} \frac{2}{n} \sum_{k=n / 2+1}^{n} \omega_{k} \omega_{2 k}=\xi \cdot p_{11} .
$$

Proposition 2.8. For $\mu$-almost all $\omega$,

$$
\lim _{n \rightarrow \infty} \frac{1}{n} \sum_{j=1}^{n} \omega_{j} \omega_{2 j}=\xi \cdot p_{11} .
$$

Proof: The proof is the same as that of Proposition 2.5 by using Corollary 2.7 and Lemma 2.2.

For $n \in \mathbb{N}$ and $\omega \in \Sigma$, denote

$$
h_{n}(\omega):=\log \mu\left(C_{2 n}(\omega)\right)-\log \mu\left(C_{n}(\omega)\right) .
$$

Lemma 2.9. For $\mu$-almost all $\omega$, as $n \rightarrow \infty$,

$$
\begin{aligned}
\frac{2}{n} h_{n}(\omega)= & p_{0} \log p_{0}+p_{1} \log p_{1}+\left(1-x_{n}(\omega)\right)\left(p_{00} \log p_{00}+p_{01} \log p_{01}\right) \\
& +x_{n}(\omega)\left(p_{10} \log p_{10}+p_{11} \log p_{11}\right)+o(1) .
\end{aligned}
$$


Proof: Following [12], for positive integers $m<n$, we write $\omega_{m}^{n}$ for the word $\omega_{m} \omega_{m+1} \cdots \omega_{n}$. For $i, j \in\{0,1\}$ and $\omega \in \Sigma$, denote

$$
N_{i}\left(\omega_{m}^{n}\right)=\sharp\left\{m \leq k \leq n: \omega_{k}=i\right\}
$$

and

$$
N_{i j}\left(\omega_{m}^{n}\right)=\sharp\left\{m \leq k \leq n: \omega_{k} \omega_{2 k}=i j\right\} .
$$

We also denote

$$
N_{i, \text { odd }}\left(\omega_{m}^{n}\right)=\sharp\left\{m \leq k \leq n: k \text { odd }, \omega_{k}=i\right\} .
$$

Then we have

$$
\frac{\mu\left(C_{2 n}(\omega)\right)}{\mu\left(C_{n}(\omega)\right)}=p_{0}^{N_{0, \text { odd }}} p_{1}^{N_{1, \text { odd }}} p_{00}^{N_{\text {o0 }}} p_{01}^{N_{01}} p_{10}^{N_{10}} p_{11}^{N_{11}},
$$

with $N_{i, \text { odd }}=N_{i, \text { odd }}\left(\omega_{n+1}^{2 n}\right)$ and $N_{i j}=N_{i j}\left(\omega_{n / 2+1}^{n}\right)$. Thus

$$
\begin{aligned}
\frac{2}{n} h_{n}(\omega)= & \frac{N_{0, \text { odd }}}{n / 2} \log p_{0}+\frac{N_{1, \text { odd }}}{n / 2} \log p_{1} \\
& +\frac{N_{00}}{n / 2} \log p_{00}+\frac{N_{01}}{n / 2} \log p_{01}+\frac{N_{10}}{n / 2} \log p_{10}+\frac{N_{11}}{n / 2} \log p_{11} .
\end{aligned}
$$

By the classical strong Law of Large Numbers, for $\mu$-almost all $\omega$, as $n \rightarrow$ $\infty$,

$$
\frac{N_{0, \text { odd }}}{n / 2}=p_{0}+o(1), \quad \frac{N_{1, \text { odd }}}{n / 2}=p_{1}+o(1) .
$$

By Lemma 2.6, for $\mu$-almost all $\omega$, as $n \rightarrow \infty$,

$$
\frac{N_{00}}{n / 2}=\left(1-x_{n}(\omega)\right) \cdot p_{00}+o(1), \quad \frac{N_{01}}{n / 2}=\left(1-x_{n}(\omega)\right) \cdot p_{01}+o(1),
$$

and

$$
\frac{N_{10}}{n / 2}=x_{n}(\omega) \cdot p_{10}+o(1), \quad \frac{N_{11}}{n / 2}=x_{n}(\omega) \cdot p_{11}+o(1) .
$$

Therefore, as $n \rightarrow \infty$,

$$
\begin{aligned}
\frac{2}{n} h_{n}(\omega)= & p_{0} \log p_{0}+p_{1} \log p_{1}+\left(1-x_{n}(\omega)\right)\left(p_{00} \log p_{00}+p_{01} \log p_{01}\right) \\
& +x_{n}(\omega)\left(p_{10} \log p_{10}+p_{11} \log p_{11}\right)+o(1) .
\end{aligned}
$$

By Lemma 2.9 and Corollary 2.4, we immediately have the following corollary.

Corollary 2.10. For $\mu$-almost all $\omega$,

$$
\begin{aligned}
\lim _{n \rightarrow \infty} \frac{2}{n} h_{n}(\omega)= & p_{0} \log p_{0}+p_{1} \log p_{1}+(1-\xi)\left(p_{00} \log p_{00}+p_{01} \log p_{01}\right) \\
& +\xi\left(p_{10} \log p_{10}+p_{11} \log p_{11}\right) .
\end{aligned}
$$


We close this section with the following proposition which gives the local entropy $h_{\mu}(\omega)$ of the measure $\mu$ for a generic sequence $\omega$.

Proposition 2.11. For $\mu$-almost all $\omega$,

$$
\begin{array}{r}
h_{\mu}(\omega)=-\frac{1}{2}\left(p_{0} \log p_{0}+p_{1} \log p_{1}+(1-\xi)\left(p_{00} \log p_{00}+p_{01} \log p_{01}\right)\right. \\
\left.+\xi\left(p_{10} \log p_{10}+p_{11} \log p_{11}\right)\right) .
\end{array}
$$

Proof: By Corollary 2.10, we need only to show that, as $n \rightarrow \infty$,

$$
\frac{\log \mu\left(C_{n}(\omega)\right)}{n}=\frac{h_{n}(\omega)}{n}+o(1) .
$$

In fact, for all $k, n \in \mathbb{N}$

$$
\frac{1}{2^{k} n} \log \mu\left(C_{2^{k} n}(\omega)\right)=\frac{1}{2^{k} n}\left(\sum_{i=1}^{k-1} h_{2^{i} n}(\omega)+\log \mu\left(C_{n}(\omega)\right)\right) .
$$

Then $\mu$-almost surely, for all $n \in \mathbb{N}$, as $k \rightarrow \infty$,

$$
\frac{1}{2^{k} n} \log \mu\left(C_{2^{k} n}(\omega)\right)=\frac{h_{n}(\omega)}{n}+o(1) .
$$

Applying Lemma 2.2, we complete the proof.

\section{Proof of Theorem 1.3}

We first prove the lower bound. We will need the measures defined in Subsection 2.4 and we will conclude by applying Billingsley's lemma for Bowen's entropy (Theorem 2.1).

Given $\alpha \in[0,1]$, let $\mu_{\alpha}$ be the probability measure on $\Sigma$ constructed in Subsection 2.4 , by using the data $\left(p_{0}, p_{1}\right):=(1 / 2,1 / 2)$ and

$$
\left(\begin{array}{ll}
p_{00} & p_{01} \\
p_{10} & p_{11}
\end{array}\right):=\left(\begin{array}{cc}
2 \alpha & 1-2 \alpha \\
1-2 \alpha & 2 \alpha
\end{array}\right)
$$

Then

$$
\mu_{\alpha}\left(C_{n}\left(\omega_{1} \cdots \omega_{n}\right)\right)=\frac{1}{2^{\lceil n / 2\rceil}} \cdot \prod_{k=1}^{\lfloor n / 2\rfloor} p_{\omega_{k} \omega_{2 k}} .
$$

We will prove that the measure $\mu_{\alpha}$ is supported on the set $\mathcal{N} \cap A_{\alpha}$.

Lemma 3.1. We have

$$
\mu_{\alpha}\left(\mathcal{N} \cap A_{\alpha}\right)=1
$$


Proof: Note that by our choice of data,

$$
\xi=\frac{p_{1}+p_{01}}{2-p_{11}+p_{01}}=\frac{\frac{1}{2}+1-2 \alpha}{2-2 \alpha+1-2 \alpha}=\frac{1}{2} .
$$

Hence by Proposition 2.8, for $\mu_{\alpha}$-almost all $\omega$,

$$
\lim _{n \rightarrow \infty} \frac{1}{n} \sum_{k=1}^{n} \omega_{k} \omega_{2 k}=\xi \cdot p_{11}=\frac{1}{2} \cdot 2 \alpha=\alpha .
$$

Thus $\mu_{\alpha}\left(A_{\alpha}\right)=1$.

Now, we show $\mu_{\alpha}(\mathcal{N})=1$. We can divide the set of natural numbers into infinitely many subsets of the form

$$
A_{k}=\left\{2 k-1,4 k-2, \ldots, 2^{\ell}(2 k-1), \ldots\right\} \quad(k \geq 1) .
$$

Let $\mathcal{B}_{k}$ be the $\sigma$-field generated by the events $\left\{\omega_{2^{\ell}(2 k-1)}=1\right\}, \ell \geq$ 0 . Observe that for the measure $\mu_{\alpha}$ the $\sigma$-fields $\mathcal{B}_{k}$ are independent. Observe further that $\mu_{\alpha}\left(\omega_{2^{\ell}(2 k-1)}=1\right)=1 / 2$ for every $k, \ell$. Indeed, for $\ell=0$, it follows from the definition of $\mu_{\alpha}$. For general $\ell$, this is proved by induction:

$$
\begin{aligned}
\mu_{\alpha}\left(\omega_{2^{\ell}(2 k-1)}=1\right)= & \mu_{\alpha}\left(\omega_{2^{\ell}(2 k-1)}=1 \wedge \omega_{2^{\ell-1}(2 k-1)}=1\right) \\
& +\mu_{\alpha}\left(\omega_{2^{\ell}(2 k-1)}=1 \wedge \omega_{2^{\ell-1}(2 k-1)}=0\right) \\
= & \mu_{\alpha}\left(\omega_{2^{\ell-1}(2 k-1)}=1\right) \cdot \mu_{\alpha}\left(\omega_{2^{\ell}(2 k-1)}=1 \mid \omega_{2^{\ell-1}(2 k-1)}=1\right) \\
& +\mu_{\alpha}\left(\omega_{2^{\ell-1}(2 k-1)}=0\right) \cdot \mu_{\alpha}\left(\omega_{2^{\ell}(2 k-1)}=1 \mid \omega_{2^{\ell-1}(2 k-1)}=0\right) \\
= & \frac{1}{2} \cdot 2 \alpha+\frac{1}{2} \cdot(1-2 \alpha)=\frac{1}{2} .
\end{aligned}
$$

Consider now, for any $n \geq 1$, the word $\omega_{m+1} \cdots \omega_{m+n}$. If $m \geq n$, then the positions $m+1, \ldots, m+n$ come all from different $A_{k}$ 's. Thus $\omega_{m+1}, \ldots, \omega_{m+n}$ are independent and each of them takes values 0,1 with probability $1 / 2$ respectively. That is, the measure $\mu$ restricted to such subset of positions is $\left(\frac{1}{2}, \frac{1}{2}\right)$-Bernoulli, and for any word $\eta \in\{0,1\}^{n}$ with $n \leq m$, the probability that we have $\omega_{m+i}=\eta_{i}$ for $i=1, \ldots, n$ equals $2^{-n}$. Thus, for a given word $\eta \in\{0,1\}^{n}$ we can divide $\mathbb{N}$ into intervals $\left[2^{j}+1,2^{j+1}\right]$, inside all except initial finitely many of them (with $j<\log _{2} n$ ), for any $\mu_{\alpha}$-generic sequence $\omega$, the frequency of appearance of $\eta$ equals $2^{-n}+O\left(2^{-j / 2} j \log j\right)$, and this means that the $\mu_{\alpha}$-generic sequence $\omega$ is normal.

Next, we will calculate the local entropy $h_{\mu_{\alpha}}(\omega)$ of the measure $\mu_{\alpha}$ for generic sequence $\omega$. We denote, for $t \in[0,1]$,

$$
H(t)=-t \log t-(1-t) \log (1-t),
$$

with the convention that $H(0)=H(1)=0$. 
Lemma 3.2. We have

$$
h_{\mu_{\alpha}}(\omega)=\frac{1}{2} \log 2+\frac{1}{2} H(2 \alpha), \quad \mu_{\alpha} \text {-a.e. }
$$

Proof: By Proposition 2.11, we have for $\mu_{\alpha}$-almost all $\omega$,

$$
\begin{aligned}
h_{\mu_{\alpha}}(\omega)=-\frac{1}{2}\left(p_{0} \log p_{0}+p_{1} \log p_{1}+(1-\xi)\left(p_{00} \log p_{00}+p_{01} \log p_{01}\right)\right. & \left.+\xi\left(p_{10} \log p_{10}+p_{11} \log p_{11}\right)\right) \\
=- & \frac{1}{2}\left(\frac{1}{2} \log \frac{1}{2}+\frac{1}{2} \log \frac{1}{2}+\left(1-\frac{1}{2}\right)(2 \alpha \log (2 \alpha)\right. \\
& \left.+(1-2 \alpha) \log (1-2 \alpha))+\frac{1}{2}((1-2 \alpha) \log (1-2 \alpha)+2 \alpha \log (2 \alpha))\right) \\
= & \frac{1}{2} \log 2+\frac{1}{2} H(2 \alpha) .
\end{aligned}
$$

Applying Theorem 2.1, by Lemmas 3.1 and 3.2, we immediately obtain

$$
h_{\text {top }}\left(\mathcal{N} \cap A_{\alpha}\right) \geq \frac{1}{2} \log 2+\frac{1}{2} H(2 \alpha) .
$$

To finish the proof of the lower bound we note that $A \subset A_{0}$ but the measure $\mu_{0}$ is actually supported on $A$, that the measure $\mu_{1 / 2}$ is supported on $B$, and that the relation $\mathcal{N} \cap B \subset A_{1 / 2}$ follows from $\frac{1}{n} \sharp\left\{n+1 \leq j \leq 2 n: \omega_{j}=\omega_{2 j}=1\right\}=\frac{1}{n} \sharp\left\{n+1 \leq j \leq 2 n: \omega_{j}=1\right\} \rightarrow \frac{1}{2}$ being satisfied for every $\omega \in \mathcal{N} \cap B$.

For the upper bound, let us first observe that

$$
\frac{1}{n} \sum_{k=1}^{n} \omega_{k} \omega_{2 k} \leq \frac{1}{n} \sum_{k=1}^{n} \omega_{k}
$$

and the right hand side converges to $1 / 2$ for every normal sequence $\omega$. Thus, the set $\mathcal{N} \cap A_{\alpha}$ is empty for all $\alpha>1 / 2$.

To continue with the case $\alpha \leq 1 / 2$, we need the following lemma.

Lemma 3.3. Let $\omega$ be a normal sequence and let $\left(n_{k}=\ell_{1}+k \ell_{2}\right)$ be an arithmetic subsequence of $\mathbb{N}$. Then $\omega$ restricted to the positions $\left(n_{k}\right)$ is normal.

Proof: The result is originally due to Wall [13]. See also Kamae [8]. 
Let us fix some $m>0$. For $N>m$ and $i=0,1, \ldots, m$, denote by $R(N, i)$ the set $\left\{2^{i}(2 k-1), k \leq 2^{N-i-1}\right\}$ (for example, $R(N, 0)$ is the set of odd numbers smaller than $\left.2^{N}\right)$. Further, let

$$
\begin{aligned}
R(N, i, I) & :=R(N-2, i), \\
R(N, i, I I) & :=R(N-1, i) \backslash R(N-2, i),
\end{aligned}
$$

and

$$
R(N, i, I I I):=R(N, i) \backslash R(N-1, i) .
$$

Note here the following obvious relations

$$
\begin{aligned}
2 R(N, i, I) & =R(N, i+1, I) \cup R(N, i+1, I I), \\
2 R(N, i, I I) & =R(N, i+1, I I I), \\
2 R(N, i, I I I) \cap R(N, i+1) & =\emptyset .
\end{aligned}
$$

We denote by $\mathcal{N}(N, m, \varepsilon)$ the set of sequences $\omega$ such that for all $n \geq$ $N$ in each $R(n, i, *), i=0, \ldots, m, * \in\{I, I I, I I I\}$ the frequency of 1 's is between $1 / 2-\varepsilon$ and $1 / 2+\varepsilon$. By Lemma 3.3,

$$
\mathcal{N} \subset \bigcap_{\varepsilon>0} \bigcap_{m=1}^{\infty} \bigcup_{N=m+1}^{\infty} \mathcal{N}(N, m, \varepsilon) .
$$

Similarly, let us denote by $A(\alpha, N, \varepsilon)$ the set of sequences $\omega$ such that for all $n \geq N$ we have

$$
\alpha-\varepsilon<2^{-n+1} \sum_{j=1}^{2^{n-1}} \omega_{j} \omega_{2 j}<\alpha+\varepsilon .
$$

Then

$$
A_{\alpha}=\bigcap_{\varepsilon>0} \bigcup_{N=1}^{\infty} A(\alpha, N, \varepsilon) .
$$

To obtain the upper bound, we will estimate from above the number of cylinders $C_{2^{N}}\left(\omega_{1}, \ldots, \omega_{2^{N}}\right)$ needed to cover the set $\mathcal{N}(N, m, \varepsilon) \cap$ $A(\alpha, N, \varepsilon)$. Let us fix $N, m, \varepsilon$. To find the cylinders $C_{2^{N}}\left(\omega_{1}, \ldots, \omega_{2^{N}}\right)$, we should determine for each position $1 \leq n \leq 2^{N}$, which value ( 0 or 1$)$, $\omega_{n}$ will take. To this end, it would be convenient that we partition the positions from 1 to $2^{N}$ into several classes according to the values of $\omega_{n}$ and those of the couple $\left(\omega_{n}, \omega_{2 n}\right)$. We will introduce the following notations concerning the number of positions in each such class. For $i=1, \ldots, m$, $k_{1}, k_{2} \in\{0,1\}$, and $* \in\{I, I I\}$, we denote

$$
X_{k_{1} k_{2}, *}^{i}(\omega):=\sharp\left\{n \in R(N, i-1, *): \omega_{n}=k_{1}, \omega_{2 n}=k_{2}\right\} .
$$


For example, $X_{01, I}^{1}(\omega)$ denotes the number of odd positions $n$ smaller than $2^{N-2}$ such that $\omega_{n}=0, \omega_{2 n}=1$. Similarly, let

$$
X_{k_{1}, *}^{i}(\omega):=\sharp\left\{n \in R(N, i, *) ; \omega_{n}=k_{1}\right\} .
$$

The following relations are obvious: for any $i$,

$$
\begin{aligned}
X_{10, I}^{i}+X_{11, I}^{i} & =X_{1, I}^{i-1}, \\
X_{00, I}^{i}+X_{01, I}^{i} & =X_{0, I}^{i-1}, \\
X_{10, I I}^{i}+X_{11, I I}^{i} & =X_{1, I I}^{i-1}, \\
X_{00, I I}^{i}+X_{01, I I}^{i} & =X_{0, I I}^{i-1}, \\
X_{01, I}^{i}+X_{11, I}^{i} & =X_{1, I}^{i}+X_{1, I I}^{i}, \\
X_{00, I}^{i}+X_{10, I}^{i} & =X_{0, I}^{i}+X_{0, I I}^{i}, \\
X_{01, I I}^{i}+X_{11, I I}^{i} & =X_{1, I I I}^{i}, \\
X_{00, I I}^{i}+X_{10, I I}^{i} & =X_{0, I I I}^{i} .
\end{aligned}
$$

Note that, for a sequence $\omega \in \mathcal{N}(N, m, \varepsilon)$, the right hand side in all relations $(3.1)-(3.8)$ are in range $2^{N-3-i} \cdot(1-\varepsilon, 1+\varepsilon)$. Thus by $(3.1)$, (3.2), (3.5), (3.6), we have

$$
\left|X_{11, I}^{i}-X_{00, I}^{i}\right| \leq \varepsilon \cdot 2^{N-1-i},
$$

and by (3.3), (3.4), (3.7), (3.8), we have

$$
\left|X_{11, I I}^{i}-X_{00, I I}^{i}\right| \leq \varepsilon \cdot 2^{N-1-i} .
$$

Note that once we know the values of the sequence

$$
\left(X_{00, I}^{i}, X_{11, I}^{i}, X_{00, I I}^{i}, X_{11, I I}^{i}\right)_{i=1}^{m},
$$

then by (3.1)-(3.4), the values of $\left(X_{0, I}^{i}, X_{1, I}^{i}, X_{0, I I}^{i}, X_{1, I I}^{i}\right)_{i=1}^{m-1}$ are also determined.

Let us start the counting of the possible $\left(\omega_{1}, \ldots, \omega_{2^{N}}\right)$. The idea is as follows: we will first describe the sequences that can appear in $\mathcal{N}(N, m, \varepsilon)$, starting with what can happen on the odd positions $2 k-1$; after that what can happen on positions of the form $2(2 k-1)$ provided that the odd positions are already decided, and so on. Finally we will go back and add another condition to our sequences to belong to $A(\alpha, N, \varepsilon)$.

Now assume that we know the values of the sequence (3.11) of number of positions. We will count how many possible $\left(\omega_{1}, \ldots, \omega_{2^{N}}\right)$ we can have, based on the information of the values of (3.11). 
The values of $\left\{\omega_{n}: n \in R(N, 0)\right\}$ can be chosen in no more than $2^{2^{N-1}}$ ways. After we have chosen $\left\{\omega_{n}: n \in R(N, i-1)\right\}$, we can choose $\left\{\omega_{n}: n \in R(N, i)\right\}$ in no more than

$$
\left(\begin{array}{c}
X_{1, I}^{i-1} \\
X_{11, I}^{i}
\end{array}\right) \cdot\left(\begin{array}{c}
X_{0, I}^{i-1} \\
X_{00, I}^{i}
\end{array}\right) \cdot\left(\begin{array}{c}
X_{1, I I}^{i-1} \\
X_{11, I I}^{i}
\end{array}\right) \cdot\left(\begin{array}{c}
X_{0, I I}^{i-1} \\
X_{00, I I}^{i}
\end{array}\right)
$$

ways. Finally, after we have chosen $\left\{\omega_{n}: n \in R(N, i)\right\}$ for all $i \leq m$, we will still have $2^{N-m-1}$ positions left, which we can choose in no more than $2^{2^{N-m-1}}$ ways.

To continue our estimation, we will use the following fact: for $k, n \in \mathbb{N}$, $0 \leq k \leq n$,

$$
\begin{aligned}
\log \left(\begin{array}{l}
n \\
k
\end{array}\right) & =n\left(-\frac{k}{n} \log \frac{k}{n}-\frac{n-k}{n} \log \frac{n-k}{n}\right)+O(\log n) \\
& =n \cdot H\left(\frac{k}{n}\right)+O(\log n),
\end{aligned}
$$

where we recall that $H(t)=-t \log t-(1-t) \log (1-t)$ is a concave analytic function on $[0,1]$.

Note that $X_{1, I}^{i-1}$ and $X_{1, I}^{i-1}$ are in the range $2^{N-3-i} \cdot(1-\varepsilon, 1+\varepsilon)$. Applying (3.12), by (3.9), we have

$$
\log \left(\begin{array}{l}
X_{1, I}^{i-1} \\
X_{11, I}^{i}
\end{array}\right)+\log \left(\begin{array}{l}
X_{0, I}^{i-1} \\
X_{00, I}^{i}
\end{array}\right)=2 \log \left(\begin{array}{c}
2^{N-3-i} \\
X_{11, I}^{i}
\end{array}\right)+2^{N-3-i} O(\varepsilon) .
$$

Similarly, by (3.10), we have

$$
\log \left(\begin{array}{c}
X_{1, I I}^{i-1} \\
X_{11, I I}^{i}
\end{array}\right)+\log \left(\begin{array}{c}
X_{0, I I}^{i-1} \\
X_{00, I I}^{i}
\end{array}\right)=2 \log \left(\begin{array}{c}
2^{N-3-i} \\
X_{11, I I}^{i}
\end{array}\right)+2^{N-3-i} O(\varepsilon) .
$$

Thus, for fixed values of the sequence (3.11), the logarithm of the total number $Z$ of cylinders needed to cover the corresponding part of $\mathcal{N}(N, m, \varepsilon)$ satisfies

$$
\begin{aligned}
& \log Z\left(\left(X_{00, I}^{i}, X_{11, I}^{i}, X_{00, I I}^{i}, X_{11, I I}^{i}\right)_{i=1}^{m}\right) \leq\left(2^{N-1}+2^{N-m-1}\right) \log 2 \\
& +\sum_{i=1}^{m}\left(2 \log \left(\begin{array}{c}
2^{N-3-i} \\
X_{11, I}^{i}
\end{array}\right)+2 \log \left(\begin{array}{c}
2^{N-3-i} \\
X_{11, I I}^{i}
\end{array}\right)+2^{N-3-i} O(\varepsilon)\right) .
\end{aligned}
$$


By (3.12), applying the Jensen inequality for the concave function $H$, we get

$$
\begin{aligned}
& \log Z\left(\left(X_{00, I}^{i}, X_{11, I}^{i}, X_{00, I I}^{i}, X_{11, I I}^{i}\right)_{i=1}^{m}\right) \leq\left(2^{N-1}+2^{N-m-1}\right) \log 2 \\
& \quad+\sum_{i=1}^{m} 2^{N-i-1} \cdot H\left(\frac{1}{\sum_{i=1}^{m} 2^{N-i-1}} \cdot \sum_{i=1}^{m} 2^{N-i-2} \frac{X_{11, I}^{i}+X_{11, I I}^{i}}{2^{N-i-3}}\right) \\
& \quad+\sum_{i=1}^{m} 2^{N-i-3} \cdot O(\varepsilon) .
\end{aligned}
$$

Hence,

$$
\begin{aligned}
\log Z\left(\left(X_{00, I}^{i}, X_{11, I}^{i}, X_{00, I I}^{i}, X_{11, I I}^{i}\right)_{i=1}^{m}\right) \\
\leq 2^{N-1} \log 2+2^{N-1} H\left(2^{-N+2} \sum_{i=1}^{m}\left(X_{11, I}^{i}+X_{11, I I}^{i}\right)\right) \\
\quad+2^{N} \cdot\left(O\left(\varepsilon+2^{-m}\right)\right) .
\end{aligned}
$$

Moreover, there are no more than

$$
\prod_{i=1}^{m} 2^{4(N-i-3)}<2^{4 m N}=o\left(2^{2^{N}}\right)
$$

possible values of $\left(X_{00, I}^{i}, X_{11, I}^{i}, X_{00, I I}^{i}, X_{11, I I}^{i}\right)_{i=1}^{m}$. We remark here that the number of possibilities is much less, because of (3.9) and (3.10). But estimate (3.14) is enough for us.

On the other hand, for all $\omega \in A(\alpha, N, \varepsilon)$,

$$
\left|2^{-N+1} \sum_{i=1}^{m}\left(X_{11, I}^{i}+X_{11, I I}^{i}\right)-\alpha\right|<\varepsilon+2^{-m} .
$$

Thus by estimates (3.13) and (3.14), the logarithm of the number of cylinders $C_{2^{N}}\left(\omega_{1}, \ldots, \omega_{2^{N}}\right)$ needed to cover the set $\mathcal{N}(N, m, \varepsilon) \cap A(\alpha, N, \varepsilon)$ is less than

$$
\begin{aligned}
& o\left(2^{N}\right)+\log Z\left(\left(X_{00, I}^{i}, X_{11, I}^{i}, X_{00, I I}^{i}, X_{11, I I}^{i}\right)_{i=1}^{m}\right) \\
& \quad \leq 2^{N-1} \log 2+2^{N-1}\left(H(2 \alpha)+O\left(\varepsilon+2^{-m}\right)\right)+2^{N} \cdot\left(O\left(\varepsilon+2^{-m}\right)\right) .
\end{aligned}
$$

Dividing the above value by $2^{N}$, and letting $m, N$ go to infinity and $\varepsilon$ to 0 , we finish the proof of the upper bound. 


\section{Proofs of Theorem 1.4 and Corollary $\mathbf{1 . 5}$}

Given $p, q \in[0,1]$, let $\mu_{p, q}$ be the probability measure on $\Sigma$ constructed in Subsection 2.4 by using the data $\left(p_{0}, p_{1}\right):=(1-p, p)$ and

$$
\left(\begin{array}{ll}
p_{00} & p_{01} \\
p_{10} & p_{11}
\end{array}\right):=\left(\begin{array}{cc}
1-p & p \\
1-q & q
\end{array}\right)
$$

The proof of Theorem 1.4 is based on the following lemmas.

Lemma 4.1. If $p=(2 \theta-\alpha) /(2-\theta)$ and $q=\alpha / \theta$, then

$$
\mu_{p, q}\left(E_{\theta} \cap A_{\alpha}\right)=1 \text {. }
$$

Proof: By Proposition 2.5, $\mu_{p, q^{-}}$-almost surely

$$
\lim _{n \rightarrow \infty} \frac{1}{n} \sum_{k=1}^{n} \omega_{k}=\xi=\frac{p_{1}+p_{01}}{2-p_{11}+p_{01}}=\frac{2 p}{2+p-q}=\theta,
$$

where the last equality comes from the choices of $p$ and $q$. Thus $\mu_{p, q}\left(E_{\theta}\right)=$ 1.

On the other hand, by Proposition 2.8, for $\mu_{p, q}$-almost all $\omega$,

$$
\lim _{n \rightarrow \infty} \frac{1}{n} \sum_{k=1}^{n} \omega_{k} \omega_{2 k}=\xi \cdot p_{11}=\theta \cdot q=\alpha .
$$

By Lemma 2.2, we conclude $\mu_{p, q}\left(A_{\alpha}\right)=1$.

Lemma 4.2. For $p=(2 \theta-\alpha) /(2-\theta)$ and $q=\alpha / \theta$, we have

$$
h_{\mu_{p, q}}(\omega)=\left(1-\frac{\theta}{2}\right) H\left(\frac{2 \theta-\alpha}{2-\theta}\right)+\frac{\theta}{2} H\left(\frac{\theta-\alpha}{\theta}\right), \quad \mu_{p, q} \text {-a.e. }
$$

Proof: By Proposition 2.11 we have, for $\mu_{p, q}$ almost all $\omega \in \Sigma$,

$$
\begin{aligned}
h_{\mu_{p, q}}(\omega)= & -\frac{1}{2}((1-p) \log (1-p)+p \log p+(1-\theta)(1-p) \log (1-p) \\
& +(1-\theta) p \log p+\theta(1-q) \log (1-q)+\theta q \log q) \\
= & \frac{1}{2}((2-\theta) H(p)+\theta H(q)) \\
= & \left(1-\frac{\theta}{2}\right) H\left(\frac{2 \theta-\alpha}{2-\theta}\right)+\frac{\theta}{2} H(\theta-\alpha \theta) .
\end{aligned}
$$

Lemma 4.3. If $\theta \notin[\alpha,(2+\alpha) / 3]$, we have $E_{\theta} \cap A_{\alpha}=\emptyset$. Otherwise, for $p=(2 \theta-\alpha) /(2-\theta)$ and $q=\alpha / \theta$, we have for all $\omega \in E_{\theta} \cap A_{\alpha}$,

$$
h_{\mu_{p, q}}(\omega)=\left(1-\frac{\theta}{2}\right) H\left(\frac{2 \theta-\alpha}{2-\theta}\right)+\frac{\theta}{2} H\left(\frac{\theta-\alpha}{\theta}\right) \text {. }
$$


Proof: Observe that, for any $\omega \in E_{\theta} \cap A_{\alpha}$, for any small $\varepsilon>0$, and for $n$ large enough, we have

$$
\begin{aligned}
& N_{1}\left(\omega_{n / 2}^{n}\right) \in\left[\frac{\theta n}{2}(1-\varepsilon), \frac{\theta n}{2}(1+\varepsilon)\right], \\
& N_{1}\left(\omega_{n}^{2 n}\right) \in[\theta n(1-\varepsilon), \theta n(1+\varepsilon)], \\
& N_{11}\left(\omega_{n}^{2 n}\right) \in\left[\frac{\alpha n}{2}(1-\varepsilon), \frac{\alpha n}{2}(1+\varepsilon)\right] .
\end{aligned}
$$

The obvious inequalities

$$
N_{11}\left(\omega_{n}^{2 n}\right) \leq N_{1}\left(\omega_{n / 2}^{n}\right)
$$

and

$$
N_{1}\left(\omega_{n}^{2 n}\right)-N_{11}\left(\omega_{n}^{2 n}\right) \leq n / 2+N_{0}\left(\omega_{n / 2}^{n}\right)=n-N_{1}\left(\omega_{n / 2}^{n}\right)
$$

imply $\theta \in[\alpha,(2+\alpha) / 3]$. Furthermore, we have

$$
\begin{aligned}
\log \mu_{p, q}( & \left.C_{2 n}(\omega)\right)-\log \mu_{p, q}\left(C_{n}(\omega)\right) \\
= & N_{11}\left(\omega_{n}^{2 n}\right) \log q+\left(N_{1}\left(\omega_{n / 2}^{n}\right)-N_{11}\left(\omega_{n}^{2 n}\right)\right) \log (1-q) \\
& +\left(N_{1}\left(\omega_{n}^{2 n}\right)-N_{11}\left(\omega_{n}^{2 n}\right)\right) \log p \\
& +\left(n-N_{1}\left(\omega_{n / 2}^{n}\right)-N_{1}\left(\omega_{n}^{2 n}\right)+N_{11}\left(\omega_{n}^{2 n}\right)\right) \log (1-p) \\
= & n\left(\left(1-\frac{\theta}{2}\right) H\left(\frac{2 \theta-\alpha}{2-\theta}\right)+\frac{\theta}{2} H\left(\frac{\theta-\alpha}{\theta}\right)+\varepsilon \cdot O(1)\right) .
\end{aligned}
$$

Hence, by the same argument as in the proof of Proposition 2.11, we have for all $\omega \in E_{\theta} \cap A_{\alpha}$,

$$
\begin{aligned}
h_{\mu_{p, q}}(\omega) & =\lim _{n \rightarrow \infty} \frac{-\log \mu_{p, q}\left(C_{n}(\omega)\right)}{n} \\
& =\left(1-\frac{\theta}{2}\right) H\left(\frac{2 \theta-\alpha}{2-\theta}\right)+\frac{\theta}{2} H\left(\frac{\theta-\alpha}{\theta}\right) .
\end{aligned}
$$

Proof of Theorem 1.4: We note that by Theorem 2.1, the lower bound of Theorem 1.4 follows from Lemmas 4.1 and 4.2 and the upper bound follows from Lemma 4.3. We thus have completed our proof.

Proof of Corollary 1.5: Note that for any $\theta, E_{\theta} \cap A_{\alpha} \subset A_{\alpha}$. Thus, if $h_{\text {top }}\left(E_{\theta} \cap A_{\alpha}\right)=h_{\text {top }}\left(A_{\alpha}\right)$ for some $\theta$, then this $\theta$ is the maximal point for the entropy formula of $h_{\mathrm{top}}\left(E_{\theta} \cap A_{\alpha}\right)$. We remark that the entropy 
formula of $h_{\text {top }}\left(E_{\theta} \cap A_{\alpha}\right)$ in Theorem 1.4 is analytic and concave with respect to the variable $\theta$ and the partial derivative

$$
\frac{\partial h_{\mathrm{top}}\left(E_{\theta} \cap A_{\alpha}\right)}{\partial \theta}=\frac{1}{2} \log \frac{\theta(2-3 \theta+\alpha)^{3}}{(2 \theta-\alpha)^{2}(\theta-\alpha)(2-\theta)}=0
$$

if and only if (1.1) holds. We then have proved the first assertion.

By Theorems 1.1 and 1.2, $h_{\text {top }}(A)=h_{\text {top }}\left(A_{0}\right)$. By Theorem 1.4, $h_{\text {top }}\left(E_{\theta} \cap A\right)=h_{\text {top }}\left(E_{\theta} \cap A_{0}\right)$. Then the second assertion follows by taking $\alpha=0$.

\section{Acknowledgements}

We thank the referees for the valuable remarks and suggestions which significantly improved the presentation of the paper. We thank one of the referees for pointing out us the reference [13] of Wall's Ph.D. thesis where we can find the original version of Lemma 3.3.

The work was supported by the France-Poland bilateral project PHC Polonium (44851YC, PPN/BFR/2019/1/00013). M. Rams was also partially supported by National Science Centre grant 2019/33/B/ST1/00275 (Poland).

\section{References}

[1] C. Aistleitner, V. Becher, and O. Carton, Normal numbers with digit dependencies, Trans. Amer. Math. Soc. 372(6) (2019), 4425-4446. DOI : 10.1090/ $\operatorname{tran} / 7706$.

[2] P. Billingsley, Hausdorff dimension in probability theory II, Illinois J. Math. 5(2) (1961), 291-298. DOI : 10.1215/ijm/1255629826.

[3] R. Bowen, Topological entropy for noncompact sets, Trans. Amer. Math. Soc. 184 (1973), 125-136. DOI : 10.2307/1996403.

[4] K. Falconer, "Fractal Geometry: Mathematical Foundations and Applications", John Wiley \& Sons, Ltd., Chichester, 1990.

[5] A.-H. FAn, L. LiaO, AND J.-H. MA, Level sets of multiple ergodic averages, Monatsh. Math. 168(1) (2012), 17-26. DOI : 10.1007/s00605-011-0358-5.

[6] A.-H. Fan, J. Schmeling, And M. Wu, Multifractal analysis of some multiple ergodic averages, Adv. Math. 295 (2016), 271-333. DOI: 10.1016/j.aim.2016. 03.012 .

[7] W. Hoeffoding, Probability inequalities for sums of bounded random variables, J. Amer. Statist. Assoc. 58(301) (1963), 13-30. DOI: 10.2307/2282952.

[8] T. Kamae, Subsequences of normal sequences, Israel J. Math. 16 (1973), 121-149. DOI : $10.1007 /$ BF02757864.

[9] R. Kenyon, Y. Peres, and B. Solomyak, Hausdorff dimension for fractals invariant under multiplicative integers, Ergodic Theory Dynam. Systems 32(5) (2012), 1567-1584. DOI : 10.1017/S0143385711000538. 
[10] Y. KIFER, A nonconventional strong law of large numbers and fractal dimensions of some multiple recurrence sets, Stoch. Dyn. 12(3) (2012), 1150023, 21 pp. DOI : $10.1142 / \mathrm{S} 0219493711500237$.

[11] J.-H. Ma And Z.-Y. Wen, A Billingsley type theorem for Bowen entropy, $C$. R. Math. Acad. Sci. Paris 346(9-10) (2008), 503-507. DOI: 10.1016/j.crma. 2008.03.010.

[12] Y. Peres andn B. Solomyak, Dimension spectrum for a nonconventional ergodic average, Real Anal. Exchange 37(2) (2011/12), 375-388.

[13] D. D. WALL, Normal numbers, Thesis (Ph.D.), University of California, Berkeley (1950).

Lingmin Liao

LAMA UMR 8050, CNRS, Université Paris-Est Créteil, 61 Avenue du Général de Gaulle, 94010 Créteil Cedex, France

E-mail address: lingmin.liao@u-pec.fr

Michał Rams

Institute of Mathematics, Polish Academy of Sciences, ul. Śniadeckich 8, 00-656 Warszawa, Poland

E-mail address: rams@impan.pl

Primera versió rebuda el 25 de setembre de 2019, darrera versió rebuda el 10 de setembre de 2020. 